\title{
Existe relação entre a prevalência de disfunção temporomandibular e a fase do curso durante a graduação?
}

Is there a relationship between the prevalence of temporomandibular joint disorder and the phase of the during graduation?

¿Existe una relación entre la prevalencia del trastorno temporomandibular

y la fase del curso durante la graduación?

Antônio Marcos de Souza PRATES ${ }^{1}$

Edilaine Soares SANTOS ${ }^{1}$

Fabiele Perpétua Chagas Sabatim BARROS ${ }^{1}$

Karolina Nunes Freitas MORAIS ${ }^{1}$

Rachel Silva LIMA ${ }^{1}$

Valthierre Nunes de LIMA ${ }^{2}$

Lamis Meorin NOGUEIRA ${ }^{3}$

${ }^{1}$ Acadêmico(a) de Graduacão em Odontologia, Centro Universitário de Rio Preto (UNIRP), 15025-400, São José do Rio Preto - SP, Brasil

${ }^{2}$ Professor Doutor, Docente das Disciplinas de Cirurgia Oral, Disfunção Temporomandibular e Dor Orofacial do Centro Universitário de Rio Preto (UNIRP), 15025-400, São José do Rio Preto - SP, Brasil

${ }^{3}$ Professora Doutora, Docente das Disciplinas de Anatomia Humana e Cirurgia Oral do Centro Universitário do Norte Paulista (UNORP), 15020-040, São José do Rio Preto - SP, Brasil

\section{Resumo}

Introdução: As desordens do complexo craniomandibular são caracterizadas por distúrbios musculoesqueléticos que estão intimamente ligados ao seu difícil diagnóstico, em virtude da etiologia multifatorial da disfunção temporomandibular. Objetivo: Averiguar a possível relação existente da prevalência de sintomatologia da disfunção temporomandibular em universitários com o período do curso, de acordo com as variáveis de grau da disfunção, período matriculado, gênero sexual e idade. Material e método: O estudo foi composto por 182 acadêmicos, na qualidade de voluntário, compreendidos do segundo ao décimo período, regularmente matriculados no $2^{\circ}$ semestre do curso de Odontologia, no ano de 2019 , em uma universidade privada de São José do Rio Preto-SP, Brasil. O questionário utilizado foi proposto pela Academia Americana de Dor Orofacial, contendo dez questões com respostas objetivas do tipo sim/não, acerca dos sintomas mais frequentes de dor orofacial e disfunção temporomandibular. O Índice Anamnésico de Fonseca foi utilizado para avaliar o grau de disfunção dos estudantes, com valor de 0 ou 10 pontos por questão, totalizando a somatória de até 100 pontos. Resultados: Os voluntários mais acometidos pela sintomatologia foram os presentes no oitavo e décimo período do curso, havendo menor prevalência nos integrantes do quarto período. Verificou-se que o gênero feminino obteve maior prevalência de sintomatologia quando comparado ao masculino, com faixa etária de 18 a 47 anos de idade. Conclusão: A prevalência da sintomatologia de disfunção temporomandibular em universitários parece ter relação à época do curso, pois os estudantes matriculados nos estágios finais do curso demonstraram maior predomínio de disfunção temporomandibular.

Descritores: Articulação Temporomandibular; Dor Facial; Síndrome da Disfunção da Articulação Temporomandibular; Transtornos da Articulação Temporomandibular.

\section{Abstract}

Introduction: Craniomandibular complex disorders are characterized by musculoskeletal disorders that are closely linked to their difficult diagnosis, due to the multifactorial etiology of temporomandibular joint disorder. Objective: To investigate the possible relationship between the prevalence of symptoms of temporomandibular joint disorder in university students and the duration of the course, according to the variables of degree of dysfunction, enrolled period, gender and age. Material and method: The study consisted of 182 students, as volunteers, from the second to the tenth period, regularly enrolled in the 2nd semester of the Dentistry course, in 2019 , at a private university in São José do Rio Preto- SP, Brazil. The questionnaire used was proposed by the American Academy of Orofacial Pain, containing ten questions with objective yes/no answers, about the most frequent symptoms of orofacial pain and temporomandibular joint disorder. The Fonseca Anamnesis Index was used to assess the degree of dysfunction of the students, with a value of 0 or 10 points per question, totaling the sum of up to 100 points. Results: The volunteers most affected by the symptoms were those present in the eighth and tenth period of the course, with a lower prevalence in the members of the fourth period. It was found that the female gender had a higher prevalence of symptoms when compared to the male, aged 18 to 47 years old. Conclusion: The prevalence of temporomandibular joint disorder symptoms in university students seems to be related to the time of the course, as students enrolled in the final stages of the course showed a greater prevalence of temporomandibular joint disorder.

Descriptors: Temporomandibular Joint; Facial Pain; Temporomandibular Joint Dysfunction Syndrome; Temporomandibular Joint Disorders.

\section{Resumen}

Introducción: los trastornos del complejo craneomandibular se caracterizan por trastornos musculoesqueléticos que están estrechamente relacionados con su difícil diagnóstico, debido a la etiología multifactorial del trastorno temporomandibular. Objetivo: investigar la posible relación entre la prevalencia de síntomas de trastorno temporomandibular en estudiantes universitarios y el período del curso, de acuerdo con las variables de grado de disfunción, período de inscripción, sexo y edad. Material y método: El estudio consistió en 182 estudiantes, como voluntarios, del segundo al décimo período, inscritos regularmente en el segundo semestre del curso de Odontología, en 2019, en una universidad privada en São José do Rio Preto- SP, Brasil. El cuestionario utilizado fue propuesto por la Academia Estadounidense de Dolor Orofacial, que contiene diez preguntas con respuestas objetivas sí/no, sobre los síntomas más frecuentes de dolor orofacial y trastorno temporomandibular. El índice de anamnesis de Fonseca se utilizó para evaluar el grado de disfunción de los estudiantes, con un valor de 0 o 10 puntos por pregunta, totalizando la suma de hasta 100 puntos. Resultados: los voluntarios más afectados por los síntomas fueron los presentes en el octavo y décimo período del curso, con una menor prevalencia en los miembros del cuarto período. Se encontró que el género femenino tenía una mayor prevalencia de síntomas en comparación con el masculino, de 18 a 47 años de edad. Conclusión: La prevalencia de los síntomas del trastorno temporomandibular en estudiantes universitarios parece estar relacionada con el momento del curso, ya que los estudiantes matriculados en las etapas finales del curso mostraron una mayor prevalencia del trastorno temporomandibular.

Descriptores: Articulación Temporomandibular; Dolor Facial; Síndrome de Disfunción de la Articulación Temporomandibular; Trastornos de la Articulación Temporomandibular.

A articulação temporomandibular (ATM), por natureza, é capaz de realizar movimentos de translação e rotação devido a sua característica bicondilar que, na mastigação, desenvolve movimentos conjuntos ao mesmo tempo, constituindo um elemento imprescindível 
ao sistema estomatognático ${ }^{1,2}$. Dessa forma, a ATM é uma articulação complexa que está rodeada de movimentações repetitivas, que podem desencadear efeitos deletérios a essa articulação. Nesse raciocínio, às desordens que acometem a ATM dão-se o nome de disfunção temporomandibular (DTM), designada também de distúrbios craniomandibulares, distúrbios da ATM, transtornos da ATM, perturbações da ATM, síndrome da disfunção da articulação temporomandibular e desordens craniomandibulares ${ }^{3}$.

Os campos que envolvem as disfunções temporomandibulares e a dor orofacial vêm sendo explorados por diversos pesquisadores na tentativa de determinar, por meio de estudos epidemiológicos, a prevalência dessas sintomatologias e o impacto na vida cotidiana dos brasileiros, assim como o perfil encontrado nestes indivíduos ${ }^{4-7}$, além de discutir as associações referentes à sintomatologia, gênero sexual e idade dos pacientes ${ }^{8}$.

À vista disso, na população em geral, estima-se que $40 \%$ a $75 \%$ detenha de, no mínimo, um sinal de DTM, como os ruídos na ATM, e ainda, 33\% apresente ao menos um sintoma, como dor na face ou até mesmo na ATM $^{9}$. A DTM constitui-se, usualmente, da causa que mais desencadeia dor crônica na face, aparecendo em qualquer idade, independente do sexo ${ }^{7}$. Assim, o sintoma mais comum apontado pelos portadores de DTM é a dor $^{1,2}$, sendo a DTM relatada como a causa mais comum de dor orofacial de origem não dental $^{10}$.

Devido à abrangência da DTM de diferentes grupos etários observa-se 0 acometimento dos indivíduos de um jeito cada vez mais precoce ${ }^{4}$. Essa concepção é ratificada pelos diversos estudos epidemiológicos verificando a prevalência de DTM em grupos de crianças $^{11-13}$, adolescentes ${ }^{12-14}$, prévestibulandos $^{15}$, universitários ${ }^{3-5}$, adultos $^{8}$ e idosos $^{16,17}$. Nos grupos citados, merece atenção os estudos transversais da prevalência de DTM em estudantes de cursinho pré-vestibular, momento pregresso à graduação, que reflete os impactos da competitividade e do estresse do pré-vestibulando na busca da vaga em universidades. Neste pensamento, alguns distúrbios emocionais, tais como pressão social e familiar tornam-se regulares no processo de diagnóstico de DTM, como também o estresse e ansiedade presentes nesse grupo ${ }^{15}$. Além disso, constata-se que estudantes do sexo feminino, alunos mais velhos e os que são reputados como tensos e nervosos possuem maior possibilidade de apresentar DTM ${ }^{12}$.
O âmbito universitário presume ser um ambiente propício para desenvolver hábitos parafuncionais, que são fatores de risco para $\mathrm{DTM}^{6,12}$, salvas as causas físicas, posturais e psicológicas, que também estão intimamente associadas à etiologia da DTM $^{5}$. Isso pode ser devido ao fato de a vida acadêmica conciliar aulas teóricas, atividades laboratoriais e atendimentos clínicos, no caso dos estudantes de Odontologia, além de provas, entrega e apresentação de trabalhos. O planejamento para o atendimento clínico diário, o primeiro contato com 0 paciente, as inseguranças durante o procedimento clínico e o medo do desconhecido podem gerar níveis aumentados de ansiedade nos acadêmicos ${ }^{18}$.

A ansiedade, pelo fato de resultar e se repercutir de comportamentos emocionais, tornam-se perceptíveis que os profissionais da saúde estejam propensos a esse distúrbio nos mais variados graus ${ }^{19}$. Assim, os profissionais da área da saúde demonstram níveis aumentados de ansiedade desde a graduação, o que está sujeito a trazer problemas já na vida universitária, como pelo desempenho, mas também com consequente risco de provocar futuras doenças ${ }^{18}$. Por si só, a ansiedade não é decisiva no desenvolvimento da DTM, porém pode predispor o indivíduo ou agravar caso ele já possua os sintomas ${ }^{10}$.

Dessarte, os estudos encontrados analisam, sobretudo, a prevalência de sinais e sintomas das disfunções temporomandibulares em estudantes universitários ${ }^{3-5,20,21}$, associando à ansiedade ${ }^{18}$ ou à qualidade de vida ${ }^{22}$. Isto posto, este estudo objetivou, diante das lacunas de informação, investigar a possível relação entre a prevalência de sintomatologia de dor orofacial e de disfunção temporomandibular, de acordo com o período em que os universitários estão matriculados, levando em consideração as variáveis de gênero, idade e grau de DTM. Baseada na literatura, a ansiedade possui relação diretamente proporcional com a $\mathrm{DTM}^{18}$. Contudo, será investigado se a ansiedade e o estresse gerados nos alunos de acordo com o decorrer dos semestres são capazes de desenvolver disfunção temporomandibular nos estudantes universitários, de tal modo que os mais acometidos sejam os discentes dos estágios finais do curso.

MATERIAL E MÉTODO

Para a execução do estudo, o projeto de pesquisa foi submetido e aprovado pelo Comitê de Ética em Pesquisa do Centro Universitário de Rio Preto - CEP/UNIRP, sob o número do parecer 3.141.244 e registro CAAE 
99682918.0.0000.5604. Trata-se de um estudo transversal de caráter subjetivo para divulgar dados relativos e absolutos, sendo um trabalho do tipo não probabilístico intencional, envolvendo 182 acadêmicos.

O estudo incluiu discentes do segundo, quarto, sexto, oitavo e décimo períodos do curso de Odontologia regularmente matriculados no segundo semestre letivo do ano de 2019, turno integral, em uma universidade privada na cidade de São José do Rio Preto-SP, Brasil. Os estudantes foram previamente informados em sala de aula, após a devida autorização dos professores, sobre os objetivos concernentes à pesquisa e todos os detalhes pertinentes. Disponibilizou-se de uma sala de aula para aplicação dos questionários, com horários predefinidos, de maneira que os alunos procurassem espontaneamente a sala, como forma de não intervir na autonomia e voluntariedade dos participantes.

Verificado o interesse do participante, o Termo de Consentimento Livre e Esclarecido (TCLE) era entregue ao aluno que, em livre e total concordância, assinava e prosseguia respondendo às perguntas do questionário. As dúvidas manifestadas foram esclarecidas por um pesquisador, permanecendo a ciência do participante da preservação e sigilo de sua identidade, bem como das informações expressas no questionário. O ciclo para a coleta de dados ocorreu-se nos meses de agosto, setembro e outubro de 2019. O questionário eleito para utilização foi o preconizado pela Academia Americana de Dor Orofacial (American Academy of Orofacial Pain) e traduzido oficialmente para a língua portuguesa por Chaves et al. ${ }^{23}$.

No que diz respeito ao índice utilizado para mensurar o grau de sintomatologia da dor orofacial e disfunção temporomandibular nos alunos, foi devidamente eleito 0 Índice Anamnésico de Fonseca, que em conformidade com Chaves et al. ${ }^{23}$ se originou nos moldes do Índice Anamnésico de Helkimo, porém com algumas modificações de maneira a obedecer ao proposto pela pesquisa.

Houve adaptação quanto às respostas e atribuição da pontuação de cada questão, sendo possíveis somente respostas sim/não, valendo 0 a cada resposta negativa, e 10 pontos em caso afirmativo, não tendo a opção "às vezes", visto que a Academia Americana de Dor Orofacial não possibilita essa opção. Sendo assim, como foi considerado o questionário da Academia Americana de Dor Orofacial, houve modificação quanto à utilização do índice para determinar o grau de DTM. Consideraram-se livres de DTM, os voluntários que, no cômputo total, somasse 0 ou 10 pontos. Os que somaram de 20 a 40 pontos foram inseridos no grupo com DTM leve. Já os que obtiveram um cálculo final de 50 ou 60 pontos, resultaram-se com DTM moderada. Os casos que decorreram com DTM severa foram aqueles com soma de 70 a 100 pontos.

A veracidade e a fidedignidade dos resultados foram obtidas por um estudo piloto anterior envolvendo todos os pesquisadores, como forma de calibrá-los para a atribuição correta da pontuação de cada questão e a soma correta dos resultados. Além disso, para padronização do estudo, cada grupo por período foi avaliado por dois pesquisadores distintos, participando um terceiro examinador em casos de diferença de resultados.

Tabela 1. Questionário preconizado pela Academia Americana de Dor Orofacial (American Academy of Orofacial Pain) ${ }^{23}$

\begin{tabular}{|c|c|c|}
\hline PERGUNTA & SIM & NÃO \\
\hline $\begin{array}{l}\text { 1- Você tem dificuldade, dor ou ambas ao abrir a boca, por } \\
\text { exemplo, ao bocejar? }\end{array}$ & ( ) & ( ) \\
\hline 2- Sua mandíbula fica "presa", "travada" ou sai do lugar? & $(\mathbf{)}$ & $(\mathrm{r})$ \\
\hline $\begin{array}{l}\text { 3- Você tem dificuldade, dor ou ambas ao mastigar, falar } \\
\text { ou usar seus maxilares? }\end{array}$ & ( ) & ( ) \\
\hline 4- Você percebe ruídos na articulação de seus maxilares? & ( ) & ( ) \\
\hline $\begin{array}{l}\text { 5- Seus maxilares ficam rígidos, apertados ou cansados } \\
\text { com regularidade? }\end{array}$ & ( ) & ( ) \\
\hline $\begin{array}{l}\text { 6- Você tem dor nas orelhas ou em volta delas, nas } \\
\text { têmporas e bochechas? }\end{array}$ & ( ) & ( ) \\
\hline $\begin{array}{l}\text { 7- Você tem cefaléia, dor no pescoço ou nos dentes com } \\
\text { frequência? }\end{array}$ & ( ) & ( ) \\
\hline $\begin{array}{l}\text { 8- Você sofreu algum trauma recente na cabeça, pescoço } \\
\text { ou maxilares? }\end{array}$ & ( ) & ( ) \\
\hline $\begin{array}{l}\begin{array}{l}\text { 9- Você percebeu alguma alteração recente na sua } \\
\text { mordida? }\end{array} \\
\end{array}$ & ( ) & ( ) \\
\hline $\begin{array}{l}\text { 10- Você fez tratamento recente para um problema não } \\
\text { explicado na articulação mandibular? }\end{array}$ & & \\
\hline
\end{tabular}

RESULTADOS

Para análise e discussão viáveis dos resultados, após a resolução das questões apresentadas, os indivíduos foram separados por período matriculado, grau de DTM, gênero e idade. Desse modo, a amostra final da pesquisa foi composta por 182 participantes, alunos do primeiro ao quinto ano, respectivos estudantes do segundo ao décimo semestre do curso de Odontologia em 2019. Dos 182 integrantes que participaram da pesquisa, 138 representavam 0 sexo feminino $(75,8 \%)$ e 44 foram os que se inseriram no sexo masculino $(24,2 \%)$.

A quantidade de respostas afirmativas para as questões apresentadas exprimiu-se de diferentes resultados, uma vez que os universitários tendem a manifestar sintomas com maior frequência às relatadas em algumas questões que em outras. Nesse sentido, o participante que assinalou apenas uma questão afirmativa, totalizando 10 pontos, embora não tenha sido classificado com nenhum grau de DTM, possuiu sua resposta englobada em um panorama geral (Figura 1), posto que seja válido suscitar as perguntas que, no decurso da pesquisa, se destacaram com os resultantes superiores. 


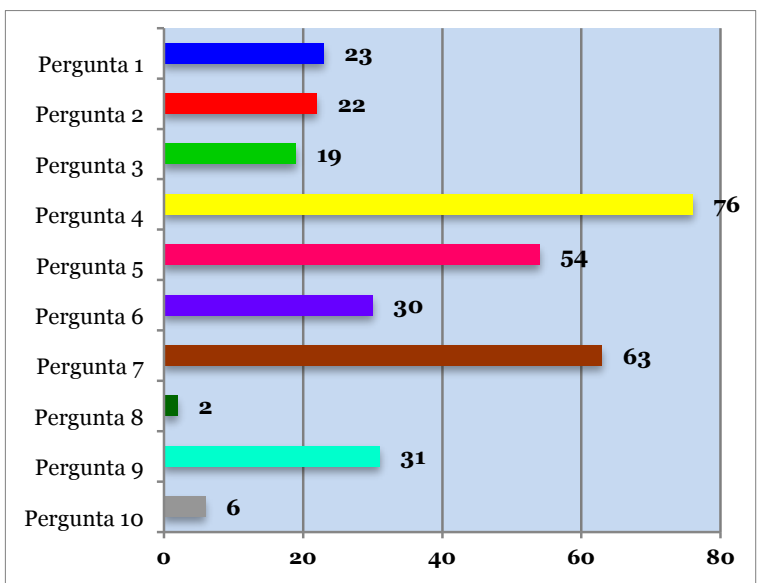

Figura 1: Gráfico com a quantidade de respostas positivas totais por pergunta.

Quanto aos indivíduos livres de DTM, com DTM leve, moderada ou severa, os resultados serão mais bem detalhados em figuras. No segundo período do curso, dos 34 participantes, mostrou-se que havia nove homens e 25 mulheres, com idades variantes dos homens entre 18 e 21 anos e das mulheres tendo de 18 a 47 anos durante a pesquisa.

Os voluntários do segundo período (figura 2), independente do sexo, em seu total, dezoito foram classificados como livres de DTM $(52,9 \%)$, doze com DTM leve $(35,2 \%)$, três com DTM moderada $(8,8 \%)$ e, por fim, um com DTM severa $(2,9 \%)$.

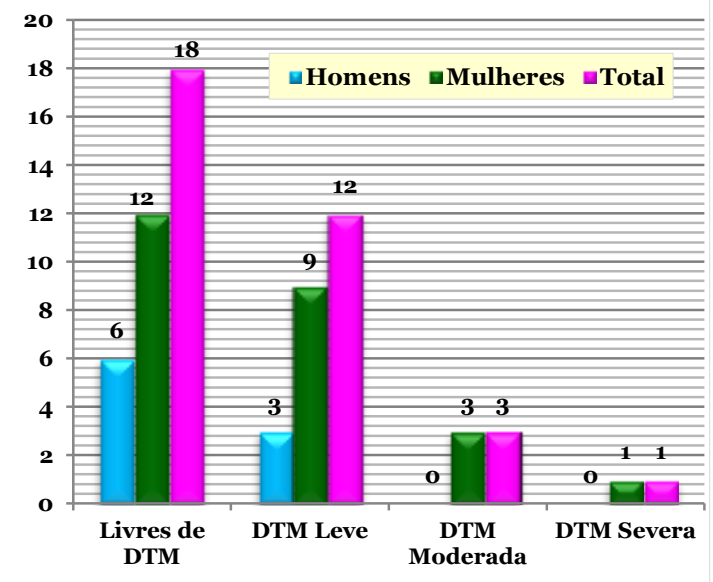

Figura 2. Gráfico dos resultados alcançados no grupo de estudantes do segundo período.

No quarto período do curso, dentre os 31 participantes, tinha doze homens e 19 mulheres, onde o sexo masculino portava idades de 18 a 24 anos e o sexo feminino com variação de idade entre 18 e 21 anos. Referente aos resultados obtidos, nessa classe, independente do sexo, os livres de DTM somavam dezenove $(61,2 \%)$, os que exibiam grau leve de DTM foram sete participantes (22,5\%), seguidos de quatro com DTM moderada (12,9\%) e, com DTM severa, apenas um indivíduo $(3,2)$. Na Figura 3, os resultados aparecem separados em homens e mulheres, o total de participantes livres de DTM e os que mostraram algum grau de DTM.

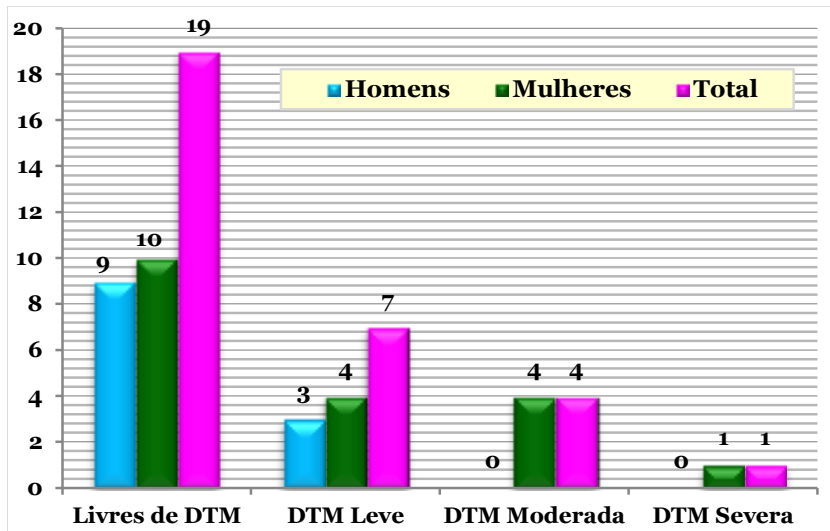

Figura 3: Gráfico dos resultados alcançados no grupo de estudantes do quarto período.

O sexto período do curso foi composto por 37 voluntários, sendo que sete eram homens e as outras 30 restantes, mulheres. A idade dos integrantes do gênero masculino era de 21 a 29 anos. Já as participantes do gênero feminino apontaram idades entre 19 e 36 anos. Nesse grupo, de acordo com a Figura 4, independente do sexo, vinte e um integrantes estavam dentre os livres de DTM (56,7\%), nove inseridos no grupo com DTM leve (24,3\%), somaram-se em quatro os com DTM moderada $(10,8 \%)$ e três com DTM severa $(8,1 \%)$.

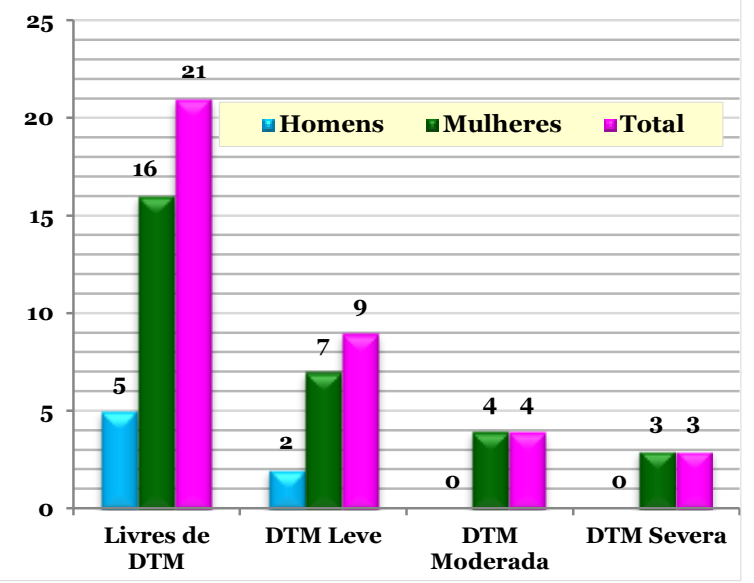

Figura 4: Gráfico dos resultados alcançados no grupo de estudantes do sexto período.

No que se refere ao oitavo período do curso, esse grupo compôs-se de 38 participantes, em um total de oito homens com idades de 21 a 28 anos e 30 mulheres, com idades variantes de 20 a 33 anos. Dentre estes estudantes, sem levar em consideração o sexo, dezoito pessoas se portaram como livres de DTM $(47,3 \%)$, dezessete com DTM leve $(44,7)$, a DTM moderada foi constatada em dois $(5,2 \%)$ e DTM severa em apenas um indivíduo $(2,6 \%)$, conforme a Figura 5. 


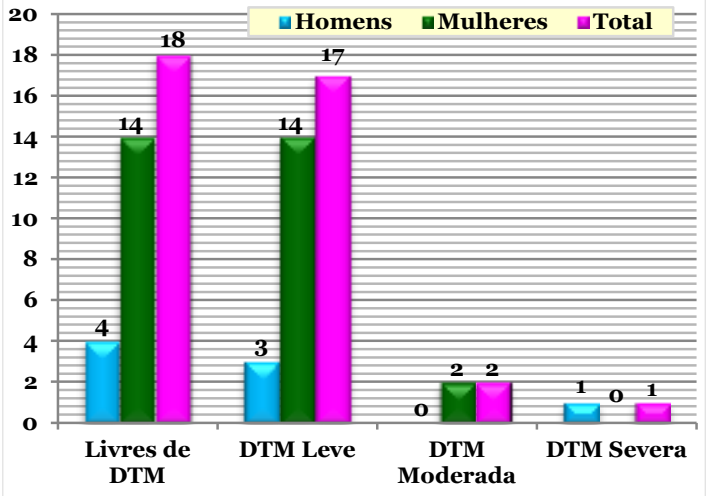

Figura 5: Gráfico dos resultados alcançados no grupo de estudantes do oitavo período.

Finalmente, 0 conjunto do décimo período do curso somou 42 integrantes, ou seja, - grupo com a maior quantidade de participantes. Destes constituintes, havia nove homens e 33 mulheres. Os homens relataram suas idades de 22 a 30 anos e as mulheres com idades entre 21 e 26 anos. Os participantes livres de DTM, segundo a Figura 6, independentes do sexo, calculavam vinte e três pessoas $(54,7 \%)$, seguidos de dezoito com DTM leve $(42,8)$, não possuindo nenhum indivíduo com grau moderado de DTM, mas tendo uma participante com DTM severa $(2,3 \%)$. No que diz respeito à somatória de alunos de todos os semestres por grau de DTM, a Figura 7 denota estes resultados, considerando o gênero e o total de portadores. Já a Figura 8 retrata o grau de DTM em função da variável de idade dos indivíduos, em geral.

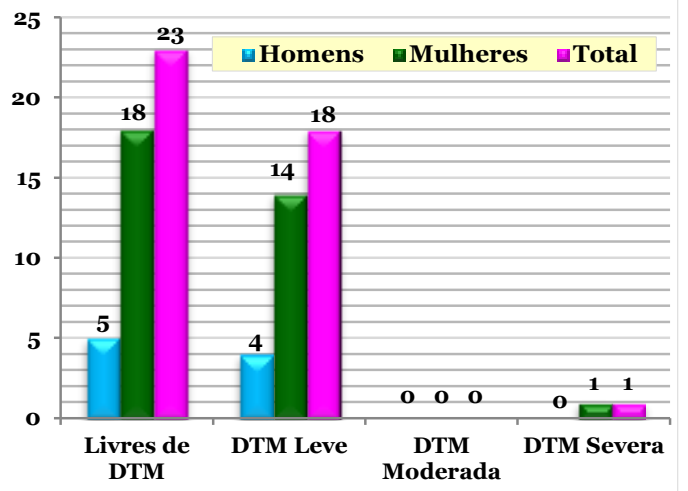

Figura 6: Gráfico dos resultados alcançados no grupo de estudantes do décimo período.

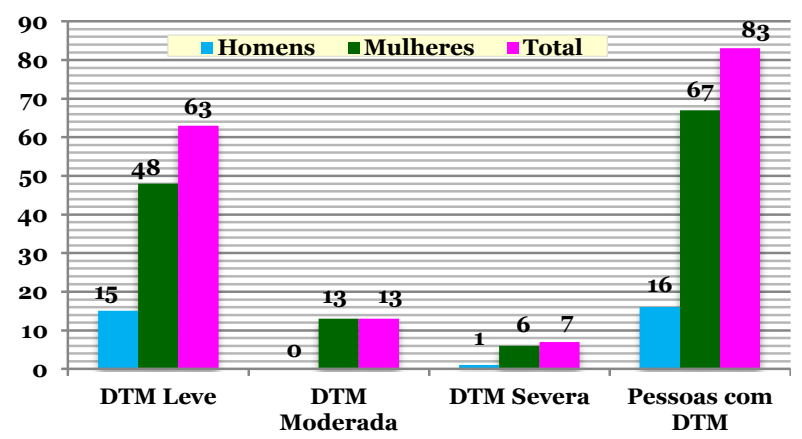

Figura 7: Gráfico do cômputo do grau de DTM em função do gênero na amostra total

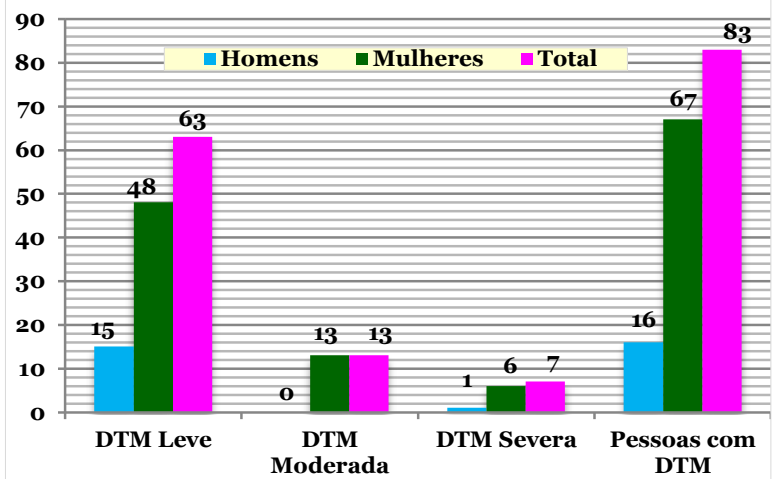

Figura 8: Gráfico da amostra total do grau de DTM em função da idade.

\section{DISCUSSÃO}

Em primeira análise, os mais afetados pela disfunção temporomandibular foram os graduandos matriculados no oitavo e décimo período do curso de Odontologia, totalizando $46,9 \%$ dos 83 portadores identificados em todos os anos. Fernandes et al. ${ }^{18}$ analisaram que a DTM acomete os alunos, independente do período cursado, porém Alves-Rezende et al. ${ }^{3}$, verificando a prevalência de DTM em universitários brasileiros, observaram maior sintomatologia em indivíduos do quinto ano e menor em integrantes do primeiro ano do curso. Logo, a época do curso e o cenário nos quais os grupos do oitavo e décimo períodos estão inseridos aparentam colaborar com os resultados obtidos, visto que no oitavo período do curso há maior quantidade de disciplinas clínicas, enquanto que no décimo, além das atividades clínicas, estão próximos de concluir a graduação.

O resultado notado nos estágios finais, atrelado ao dos períodos iniciais do curso, presume corroborar com a relação existente entre 0 predomínio da disfunção temporomandibular e o semestre em que o aluno está matriculado, uma vez que o grupo do segundo período teve $19,2 \%$ dos indivíduos com DTM e o do quarto deteve de $14,4 \%$ da amostra dos portadores de DTM. Embora tenha ocorrido uma queda do segundo para o quarto período, sob o ponto de vista do total de participantes em cada grupo, constata-se que o quarto período do curso contou com menos voluntários que o segundo.

Em segunda avaliação, dentre os participantes da pesquisa, $54,4 \%$ dos 182 participantes não foram detectados com DTM, sendo estes livres de DTM. Nesse tocante, a literatura, avaliando universitários, revela estudos que também apontam maior quantidade de voluntários sem DTM ${ }^{21}$. No entanto, outros estudos encontraram, como o de Bezerra et al. ${ }^{4}$ e o de Oliveira et al. $^{21}$, maior quantidade de 
pessoas com DTM. A prevalência dos portadores de DTM para o segundo, sexto, oitavo e décimo período do curso obteve quantidade progressiva com valores entre $43,2 \%$ e $52,6 \%$. Contudo, no estudo de Fernandes et al. $^{18}$, apenas o predomínio de DTM leve mostrou-se constante, em valores de $50 \%$ a $58 \%$ para todos os períodos. Essa discrepância pode ser devida à diferença da quantidade de estudantes participantes em cada um dos estudos e indivíduos inseridos por grupo, principalmente no quarto período que contou com menos integrantes.

De outra forma, dos 45,6\% que resultaram com DTM, 75,9\% mostrou DTM leve, moderada em $15,7 \%$ e os integrantes com DTM severa somavam-se em $8,4 \%$. Bezerra et al. ${ }^{4}$ também obtiveram resultados decrescentes para os graus de DTM leve, moderada e severa. Os altos índices de indivíduos com DTM leve, quando comparados aos graus de DTM moderada e severa, também foram bastante enfatizados pela literatura ${ }^{4,20,21}$.

Por outro lado, o levantamento de dados, por meio da aplicação de questionários que classificam somente respostas objetivas, sendo elas negativas ou positivas, apesar de serem instrumentos de avaliação breves e compreensíveis, há um risco considerável, onde pequena quantidade de manifestações clínicas que podem caracterizar uma DTM severa seja mensurada como leve ou moderada ${ }^{20}$. Da mesma maneira, pode ocorrer a situação onde o participante reporte vários sintomas considerados de DTM leve e ser inserido no grupo de DTM severa ${ }^{20}$. Por isso, tal ferramenta não pode ser a única considerada para conferir o diagnóstico3, surtindo efeitos significativos apenas na pré-triagem da disfunção temporomandibular, sobretudo, pela total incapacidade de atribuir a gravidade e 0 diagnóstico preciso em casos remotos.

Em terceira inspeção, estudos que avaliam a DTM, caracterizam-na como uma disfunção que acomete o sexo feminino com maior predileção, podendo ocorrer numa proporção de $4: 17^{10,20}$, relacionando a quantidade de $80 \%$ de mulheres em comparação a $20 \%$ de homens. Neste raciocínio, este estudo corrobora com o exposto pela literatura, uma vez que os resultados demonstraram o sexo feminino com maiores indícios de DTM, sendo que a cada cinco pessoas identificadas com DTM, apenas uma era do sexo masculino e as outras quatro, mulheres. Dentre os 83 voluntários que foram classificados com DTM havia 67 mulheres e 16 homens, com percentual de $80,7 \%$ de mulheres diante dos homens que era $19,3 \%$. Conquanto, ressalta-se que a quantidade de participantes do gênero feminino também foi maior que os do masculino, em uma razão aproximada de três.

$\mathrm{O}$ acometimento de mulheres, conforme dados da literatura, está ligado ao fato de as mulheres terem uma preocupação maior com a saúde, procurando atendimento e tratamento com uma determinada assiduidade, quando emparelhadas aos indivíduos do sexo masculino ${ }^{20}$. Além disso, as mulheres prestam mais atenção nas perguntas, respondendo positivamente maior quantidade por possuírem mais cuidado com a saúde, no geral, que os homens $^{21}$. Todavia, há relatos de que as pessoas do sexo feminino têm maior capacidade de detectar sintomatologias dolorosas, além de a articulação temporomandibular, nas mulheres, apresentar maior tendência às disfunções ${ }^{20}$. Ademais, o sexo feminino detém certas peculiaridades relacionadas ao fisiologismo hormonal, tangente a variações que ocorrem no organismo, a nível tecidual ou até mesmo estrutural, porém necessitando de mais estudos sobre o assunto $^{10,20}$.

Com relação à faixa etária dos estudantes analisados, dentre os que apresentaram algum grau de DTM, a maioria dos indivíduos $(20,5 \%)$ possuíam 20 anos e $19,3 \%$ tinha 21 anos completos na data do preenchimento do questionário. Os voluntários com idades de 22 e 23 anos, tiveram 14,4\% cada, de participantes com algum grau de DTM. Alves-Rezende et al. $^{3}$ observaram maior incidência de DTM para as idades de 21 e 22 anos. Assim, o que foi obtido pelo estudo encontra-se nos limites expressos pela literatura.

Por fim, pela característica deste estudo de não expor a causalidade e consequência da relação entre a prevalência de DTM e período de matrícula do estudante na graduação, não são permitidas conclusões definitivamente concretas sobre o assunto, transparecendo apenas dados alusivos ao problema, carecendo, por isso, de estudos mais detalhados no futuro.

CONCLUSÃO

Infere-se, portanto, baseado nos objetivos, materiais e métodos empregados e à luz dos resultados e discussão apresentados, que: 1) A prevalência da sintomatologia de dor orofacial e DTM aparentam estar relacionadas à fase do curso, pois os estudantes matriculados no oitavo e décimo períodos exibiram maior predomínio de DTM que o segundo e o quarto período; 2) A ansiedade e o estresse causados nos alunos ao decorrer dos períodos parecem 
estar vinculados à prevalência de disfunção temporomandibular nos universitários, principalmente nas etapas terminais do curso; 3 ) Em cada um dos períodos examinados, obtiveram-se maiores resultados de DTM leve e, em ordem decrescente, DTM leve, moderada e severa, com exceção do décimo período que não teve DTM moderada; 4) No que tange à variável de gênero, há o maior acometimento do gênero feminino, com valores em taxas aproximadas de $80 \%$ mulheres e $20 \%$ homens, junto à proporção de $4: 1$; 5) Em relação à variável de idade, os indivíduos mais acometidos pela DTM possuíam, na maioria das vezes, 20 e 21 anos de idade.

\section{REFERÊNCIAS}

1. Donnarumma MDC, Muzilli CA, Ferreira C, Nemr K. Disfunções Temporomandibulares: sinais, sintomas e abordagem multidisciplinar. Rev CEFAC. 2010; 12(5):788-94.

2. Pereira KNF, Andrade LLS, Costa MLG, Portal TF. Sinais e sintomas de pacientes com disfunção temporomandibular. Rev CEFAC. 2005;7(2):221-28.

3. Alves-Rezende MCR, Silva JS, Soares BMS, Bertoz FA, Oliveira DTN, Claro APRA. Estudo da prevalência de sintomatologia temporomandibular em universitários brasileiros de odontologia. Rev Odontol Araçatuba. 2009;30(1):9-14.

4. Bezerra BPN, Ribeiro AIAM, Farias ABL, Farias $A B L$, Fontes LBC, Nascimento $S R$, et al. Prevalência da disfunção temporomandibular e de diferentes níveis de ansiedade em estudantes universitários. Rev DOR. 2012;13(3):235-42.

5. Goyatá FR, Taira NV, Almeida S, Silva DM, Taira CV. Avaiação de sinais e sintomas de disfunção temporomabndibular entre acadêmicos do curso de Odontologia da Universidade Severino Sombra, Vassouras-RJ. Int J Dent. 2010;9(4):181-86.

6. Figueiredo VMG, Cavalcanti AL, Farias ABL, Nascimento SR. Prevalência de sinais, sintomas e fatores associados em portadores de disfunção temporomandibular. Acta Sci Health Sci. 2009;31(2):159-63.

7. Portinho CP, Collares MVM, Faller GJ, Fraga MM, Pinto RA. Perfil dos pacientes com disfunção temporomandibular. ACM arq catarin med. 2012;41(1):95-9.

8. Ferreira CLP, Da Silva MAMR, Felício CM. Sinais e sintomas de desordem temporomandibular em mulheres e homens. CoDAS. 2016;28(1):17-21.

9. Carrara SV, Conti PCR, Barbosa JS. Termo do 10 Consenso em Disfunção Temporomandibular e Dor Orofacial. Dental Press J Orthod. 2010;15(3):114-20.
10.Daher CRM, Cunha LF, Ferreira APL, Souza AISO, Rêgo TAM, Araújo MGR, et al. Limiar de dor, qualidade de sono e níveis de ansiedade em indivíduos com disfunção temporomandibular. Rev CEFAC. 2018;20(4): 450-58.

11.Sena MF, De Mesquita KSF, Santos FRR, Silva FWGP, Serrano KVD. Prevalência de disfunção temporomandibular em crianças e adolescentes. Rev Paul Pedriatr. 2013;31(4): 538-45.

12. Minghelli B, Cardoso I, Porfírio M, Gonçalves R, Cascalheiro S, Barreto V, et al. Prevalence of temporomandibular disorder in children and adolescents from public schools in southern Portugal. N Am J Med Sci. 2014;6(3):126-32.

13. Deng YM, Fu MK, Hägg U. Prevalence of temporomandibular joint dysfunction (TMJD) in Chinese children and adolescents. A crosssectional epidemiological study. Eur J Orthod. 1995;17(4):305-9.

14. Winocur E, Gavish A, Finkelshtein T, Halachmi M, Gazit E. Oral habits among adolescent girls and their association with symptoms of temporomandibular disorders. J Oral Rehabil. $2001 ; 28(7): 624-9$.

15. Paulino MR, Moreira VG, Lemos GA, Da Silva PLP, Bonan PRF, Batista AUD. Prevalência de sinais e sintomas de disfunção temporomandibular em estudantes prévestibulandos: associação de fatores emocionais, hábitos parafuncionais e impacto na qualidade de vida. Ciên Saúde Colet. 2018; 23(1):173-86.

16. Schmitter M, Rammelsberg $P$, Hassel $A$. The prevalence of signs and symptoms of temporomandibular disorders in very old subjects. J Oral Rehabil. 2005;32(7):467-73.

17. Cavalcanti MOA, Lima CMC, Lima JMC, Gomes I, Gordim JR. Estud Interdiscipl Envelhec. 2015;20(2):551-66.

18. Fernandes AUR, Garcia AR, Zuim PRJ, Cunha LDP, Marchiori AV. Desordem temporomandibular e ansiedade em graduandos de odontologia. Cien Odontol Bras. 2007;10(1):70-7.

19. Oliveira KL, Almeida GA, Lelis ER, Tavares M, Fernandes Neto AJ. Temporomandibular disorder and anxiety, quality of sleep, and quality of life in nursing profissionals. Braz Oral Res. 2015;29(1):1-7.

20. Medeiros SP, Batista AUD, Forte FDS. Prevalência de sintomas de disfunção temporomandibular e hábitos parafuncionais em estudantes universitários. Rev Gaúch Odontol. 201;59(2):201-8.

21. Oliveira AS, Dias EM, Contato RG, Berzin F. Prevalence study of signs and symptoms of temporomandibular disorder in Brazilian college students. Braz Oral Res. 2006;20(1):3-7. 
22. Scheffer CAC, Frigo LF. Relação da incidência de sintomas de disfunção temporomandibular com a qualidade de vida em estudantes universitários. Fisioter Bras. 2012;13(6):446-51.

23. Chaves TC, Oliveira AS, Grossi DB. Principais instrumentos para avaliação da disfunção temporomandibular; parte l: índices e questionários; uma contribuição para a prática clínica e de pesquisa. Fisioter Pesqui. 2008; 15(1):92-100.

\section{CONFLITO DE INTERESSES}

Os autores declaram não haver conflitos de interesse

AUTOR PARA CORRESPONDÊNCIA

Antônio Marcos de Souza Prates

Centro Universitário de Rio Preto (UNIRP),

15025-400, São José do Rio Preto - SP, Brasil

E-mail: prates.ams@hotmail.com

Submetido em 07/05/2020

Aceito em 23/10/2020 